\title{
Feature Detection on 3D Face Surfaces for Pose Normalisation and Recognition
}

\author{
Chris Maes, Thomas Fabry, Johannes Keustermans, Dirk Smeets, Paul Suetens and Dirk Vandermeulen
}

\begin{abstract}
This paper presents a SIFT algorithm adapted for 3D surfaces (called meshSIFT) and its applications to 3D face pose normalisation and recognition. The algorithm allows reliable detection of scale space extrema as local feature locations. The scale space contains the mean curvature in each vertex on different smoothed versions of the input mesh. The meshSIFT algorithm then describes the neighbourhood of every scale space extremum in a feature vector consisting of concatenated histograms of shape indices and slant angles. The feature vectors are reliably matched by comparing the angle in feature space.

Using RANSAC, the best rigid transformation can be estimated based on the matched features leading to $84 \%$ correct pose normalisation of $3 \mathrm{D}$ faces from the Bosphorus database. Matches are mostly found between two face surfaces of the same person, allowing the algorithm to be used for $3 \mathrm{D}$ face recognition. Simply counting the number of matches allows $93.7 \%$ correct identification for face surfaces in the Bosphorus database and $97.7 \%$ when only frontal images are considered. In the verification scenario, we obtain an equal error rate of $15.0 \%$ to $5.1 \%$ (depending on the investigated face surfaces). These results outperform most other algorithms found in literature.
\end{abstract}

\section{INTRODUCTION}

Reliable feature detection and matching on $3 D$ face surfaces is useful for several biometrics related applications, such as pose normalisation, face recognition and modeling of face deformation. In this paper, we develop a feature detection and description method for meshes, based on the SIFT algorithm [1]. This algorithm, which we call meshSIFT, allows for accurate detection of scale space extrema on meshes and a rich description of the local feature neighbourhood of the detected scale-space extrema. In this paper, we validate the meshSIFT algorithm for pose normalisation and 3D face recognition. The experiments will be performed on an extensive database of 3D faces: the Bosphorus database [2] .

The remainder of this section gives a brief overview of related work. In section II, we describe the newly developed meshSIFT algorithm for detection of scale space extrema and construction of local feature descriptors. In section III, the meshSIFT algorithm is tested on pose normalisation of 3D face scans and 3D-3D face recognition. Section IV discusses the performance of the developed algorithm. Section V, finally, concludes the paper and gives some directions for future work.

The authors are with the Faculty of Engineering, Department of Electrical Engineering, Katholieke Universiteit Leuven, Kasteelpark Arenberg 10, B-3001 Heverlee (Leuven), Belgium and Medical Imaging Center, University Hospitals Gasthuisberg, Herestraat 49 - bus 7003, B-3000 Leuven, Belgium (e-mail: thomas.fabry@uz.kuleuven.be, johannes.keustermans@uz.kuleuven.be, dirk.smeets@uz.kuleuven.be)
The Scale Invariant Feature Transform (SIFT), proposed in [1], has been shown to be a very useful methodology in 2D computer vision, where it has been used for all kinds of general object recognition tasks [1], [3], including 2D face recognition [4], [5]. Applied to three dimensions, local features like the ones used in the SIFT algorithm could be very useful for shape registration, motion capture, computer animation synthesis, object and face recognition and shape retrieval, among others.

Triggered by the success of SIFT in 2D computer vision, there have been several attempts to extend the algorithm to three dimensions. N-SIFT [6] and VSIFT [7] are extensions of SIFT for 3D volumetric (medical) image data, but also extensions of the original SIFT algorithm to work on 3D surface data have been proposed. In [8] the SIFT-algorithm is adapted to range images but is not suited for full 3D surface representations such as point clouds or meshes. The developed algorithm is hence called 2.5D SIFT.

Both [9] and [10] describe a method for detection of scale space extrema on meshes. In [9], the 3D features are detected and constructed using a general framework of scalar fields defined on 2D manifolds. As such, the features can be based on any scalar function defined over the 2D manifold, such as photometric data, mean surface curvature,... This is a strength of the algorithm, but on the other hand, the 3D geometry is in this way not directly encoded in the feature descriptor. The feature point detector is called $M e s h D o G$, and is a generalisation of the Difference of Gaussians (DoG) operator. It builds a scale space of the scalar function on the mesh, making use of the DoG operator to approximate the Laplacian operator. The feature descriptor is called MeshHOG, and is a generalisation of the histogram of oriented gradients (HOG) descriptor. The features were validated using five rigidly and non-rigidly deformed meshes.

In [10], a similar concept is developed. Here, a so-called geodesic scale space of the scalar field defined on the mesh is constructed, in which the feature points are detected. Unlike [9], an extra fine tuning step to locate the scale space extrema more precisely, eliminating groove and ridge responses, is added. The feature descriptors are based on spin images [11] and are called scale invariant spin images. The resulting algorithm is applied to face matching with expressions as well as surface alignment and stitching.

Castellani et al. [12] also describe a method for detection of scale space extrema on meshes. In contrast to [9] and [10], the scale space for feature detection is not constructed based on a scalar function on the mesh, but based on the mesh vertex locations itself. As such, the feature location 
detection of this research shows the closest resemblance to our proposed method. The feature description and matching are obtained using a Hidden Markov Model (HMM). The algorithm is validated for mesh matching on 8 models.

For years, automatic face recognition has been an active research topic. The main quest has been the detection and recognition of human faces in 2D still images and (surveillance camera) videos. A good survey hereof is presented in [13]. The SIFT-methodology has been applied for tackling this task [4], [5]. Recently, face recognition research has started to take 3D information into account when dealing with this problem. This way, it is believed that face recognition will be more robust, as 3D face models are, if accurately acquired, invariant to differences in pose and lighting conditions. This is very important for realistic situations, where face images are usually captured in environments where no fixed pose can be enforced, and also lighting conditions vary. An overview of 3D face recognition approaches can be found in [14]. Although 3D face recognition methods that make use of local features exist [15], [16], 3D face recognition using automatically located local features based on solely the mesh geometry are not commonly used in 3D face recognition.

\section{MEShSIFT}

Similar to the SIFT algorithm [1], the meshSIFT algorithm consists of three major components: keypoint detection, orientation assignment and the local feature descriptor. This section presents each of these components in detail.

\section{A. Keypoint detection}

This component identifies salient points on the mesh. Similar to the SIFT algorithm [1] a scale space approach is pursued. In this scale space salient points are detected, leading to a scale invariant descriptor. First, a scale space is constructed containing smoothed versions of the input mesh. These smoothed versions are obtained by approximating a Gaussian filter for meshes. This approximation consists of subsequent convolutions of the mesh with a binomial filter [17]. The scale space is constructed as follows:

$$
\begin{gathered}
M_{i} \quad= \begin{cases}M & \mathrm{i}=0 \\
\widehat{G}_{\sigma_{i}} \otimes M & \text { else },\end{cases} \\
\widehat{D o G}_{i}=M_{i}-M_{i-1}=\left(\widehat{G}_{\sigma_{i}}-\widehat{G}_{\sigma_{i-1}}\right) \otimes M,
\end{gathered}
$$

where $M$ is the original mesh, $\widehat{G}_{\sigma_{i}}$ and $\widehat{D o G}_{i}$ are the approximations of the Gaussian and the difference of Gaussians respectively. The standard deviation of the Gaussian filter $\widehat{G}_{\sigma_{i}}$ that was approximated to obtain a smoothed mesh $M_{i}$ is given by $\sigma_{i}$. Figure 1 shows three smoothed meshes of the constructed scale space.

Next, for the detection of salient points in the scale space, the mean curvature

$$
H(x, y)=\frac{\left(1+f_{y}^{2}\right) f_{x x}-2 f_{x} f_{y} f_{x y}+\left(1+f_{x}^{2}\right) f_{y y}}{2\left(1+f_{x}^{2}+f_{y}^{2}\right)^{\left(\frac{3}{2}\right)}}
$$

is computed for each vertex and at each scale in the scale space (with $f_{x}=\frac{\partial f(x, y)}{\partial x}, f_{x x}=\frac{\partial^{2} f(x, y)}{\partial x^{2}}$, etc). Let $H_{i}$ be

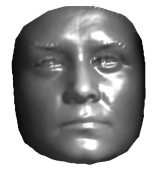

(a) $\sigma_{i}=1.83 \mathrm{~mm}$

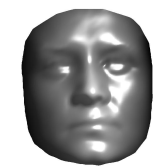

(b) $\sigma_{i}=3.66 \mathrm{~mm}$

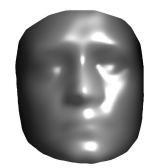

(c) $\sigma_{i}=6.18 \mathrm{~mm}$
Fig. 1: Smoothed meshes in the scale space at three different scales defined by $\sigma_{i}$.

a set containing the mean curvature for each vertex at scale $i$. Differences between subsequent scales are computed as follows:

$$
D H_{i}=H_{i+1}-H_{i} .
$$

In order to detect scale space extrema, the value $D H$ at each vertex is compared to those of its neighbors, on the same scale as well as on the upper and lower scale (see figure 2). A vertex is selected only if its value for $D H$ is larger or smaller than all of its neighbors.

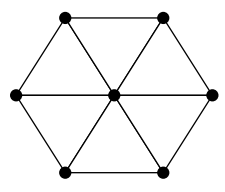

(a) $D H_{i-1}$

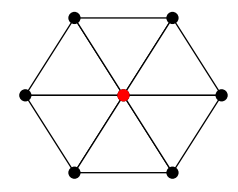

(b) $D H_{i}$

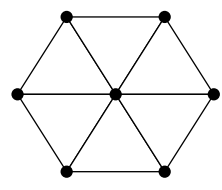

(c) $D H_{i+1}$
Fig. 2: The neighborhood of a vertex in scale space.

Finally, the correct scale $\sigma_{i}$ is assigned to each scale space extremum, leading to a keypoint with an assigned scale.

\section{B. Orientation assignment}

In order to have an orientation-invariant descriptor, each keypoint is assigned a canonical orientation. By expressing all parameters in function of the scale $\sigma_{i}$, we ensure a scale invariant descriptor as well.

Only vertices within a spherical region with radius $9 \cdot \sigma_{i}$ around each keypoint are considered. First, for each vertex within this region, the normal vector is computed (using [18]) and the geodesic distance to the respective keypoint (using [19]) is determined. Next, as shown in figure 3, all calculated normal vectors are projected onto the tangent plane to the mesh $M_{i}$ containing the keypoint.

These projected normal vectors are gathered in a weighted histogram comprising 360 bins (thus covering 360 degrees with a bin width of 1 degree). Each histogram entry is Gaussian weighted $\left(\sigma=4.5 \cdot \sigma_{i}\right)$ with its geodesic distance to the keypoint. As such, every bin represents a possible canonical orientation in the tangent plane. The resulting histogram is smoothed by convolving it three times with a Gaussian filter (17 bins, $\sigma=17$ ) for a more accurate and robust localization of the canonical orientation. Finally, the highest peak in the histogram and every peak above $80 \%$ of this highest peak value is selected as a canonical orientation. If more than one canonical orientation exists for a keypoint, this results in multiple keypoints, each assigned one of the canonical orientations. 


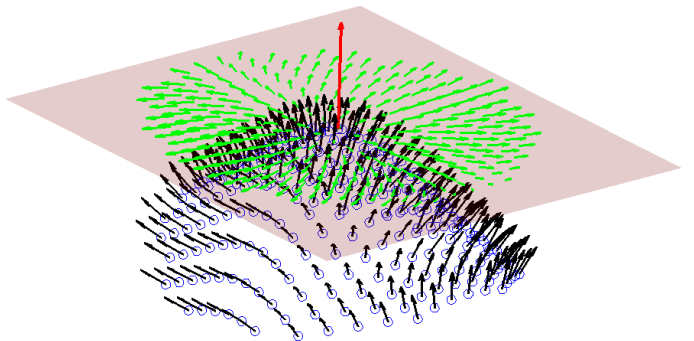

Fig. 3: The neighborhood of a scale space extremum with normals and projected normals.

\section{Local descriptor}

The local descriptor provides for each keypoint (with assigned scale and canonical orientation) a feature vector consisting of a series of concatenated histograms. Each of these histograms is calculated over a small circular region. Figure 4 shows the arrangement of these regions around the keypoint. The regions each have a geodesic radius of $3.75 \cdot \sigma_{i}$ and their centers are located at a geodesic distance of $4.5 \cdot \sigma_{i}$ (horizontal and vertical) or $4.5 \cdot \sqrt{(2)} \cdot \sigma_{i}$ (diagonal), respectively, to the keypoint.

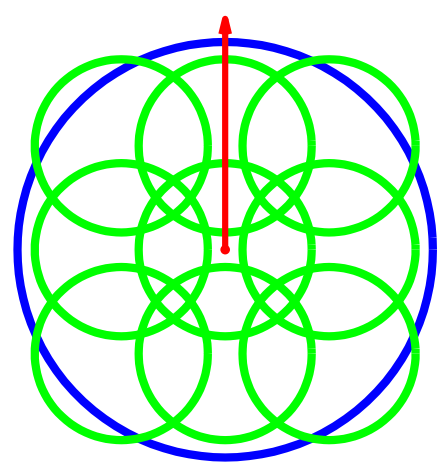

Fig. 4: Size and location of the regions w.r.t. the canonical orientation (red), used for the construction of the feature vector.

In each region (green circles in figure 4) we calculate two histograms with 8 bins each: one containing the shape index,

$$
S(x, y)=\frac{2}{\pi} \tan ^{-1}\left[\frac{k_{1}(x, y)+k_{2}(x, y)}{k_{1}(x, y)-k_{2}(x, y)}\right],
$$

(with $k_{1}$ and $k_{2}$ the maximal and minimal curvature respectively), and the other containing the slant angles, being the angle between every projected normal and the canonical orientation. First, each entry for both histograms is Gaussian weighted with the geodesic distance to the keypoint ( $\sigma=$ 4.5 $\sigma_{i}$ ) and Gaussian weighted with the geodesic distance to the center of the region (green circles in figure $4, \sigma=4.5 \cdot \sigma_{i}$ ). Next, every histogram is normalized and clipped to $\frac{1}{\sqrt{n}}$ (with $n$ the number of bins, equal to 8 in this case), reducing the influence of large histogram values. In a final step all histograms are concatenated as follows:

$$
\begin{aligned}
\widehat{F V}_{i} & =\left(\widehat{\text { hist }}_{S}\right)\left(\widehat{\text { hist }}_{\text {slant angles }}\right), i=1, . ., 9 \\
F V & =\left(\widehat{F V}_{1}, \widehat{F V}_{2}, \ldots, \widehat{F V}_{9}\right)
\end{aligned}
$$

with $F V$ the final feature vector.

\section{Matches}

In order to find corresponding keypoints, two sets of feature vectors are compared. The angle between each pair of feature vectors is calculated as follows:

$$
\alpha=\cos ^{-1}\left(\frac{<K V_{i}, K V_{j}>}{\left\|K V_{i}\right\|\left\|K V_{j}\right\|}\right) .
$$

The angles of all candidates are ranked in ascending order. If the ratio between the first and the second is smaller than 0.8 , a match is accepted; other matches are rejected. This ratio was determined emperically, and is the same as in the original SIFT algorithm [20]. Figure 5 shows two pictures of textures beloning to 3D face scans from the Bosphorus database [2] with detected keypoints and the detected matches (all calculations are performed on the meshes, the pictures are only used to show the results). Figure 6 shows a detailed view with the corresponding scale space extrema.

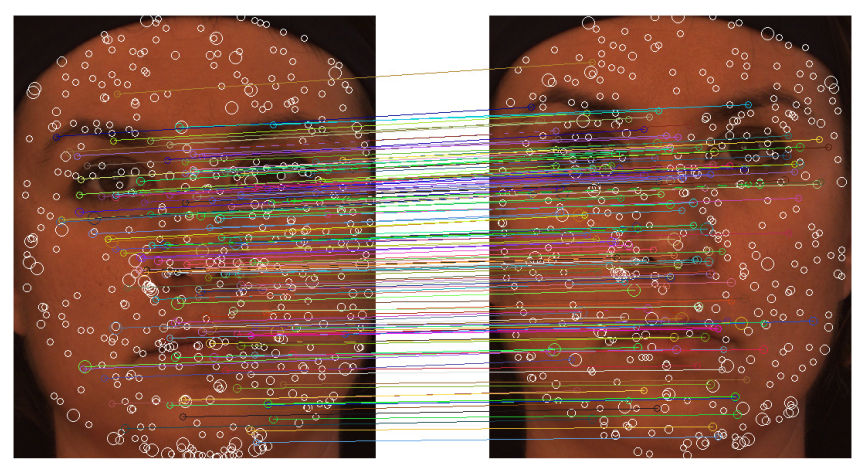

Fig. 5: Corresponding scale space extrema for two face surfaces with a neutral expression (both from the same person)

\section{APPLICATIONS AND RESULTS}

All experiments in this paper are performed on the Bosphorus 3D face database [2] ${ }^{1}$, a multi-expression and multi-pose 3D face database. The database contains 4666 textured depth maps of 105 persons in various poses, expressions and occlusion conditions, and for each depth map a subset of 24 manually labelled facial landmarks. The depth maps are converted to surface meshes in a straightforward manner.

\footnotetext{
${ }^{1}$ Available via http://bosphorus.ee.boun.edu.tr/.
} 


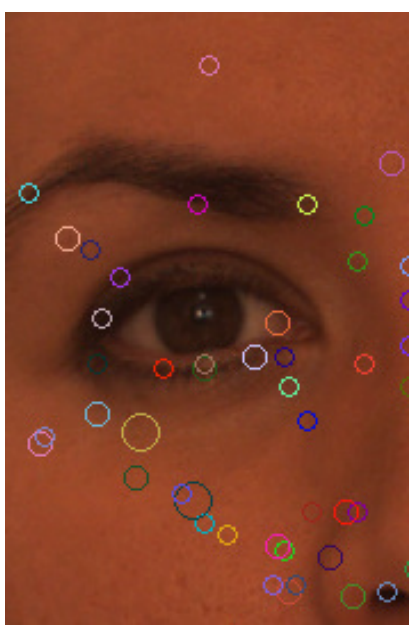

(a) Detailed view of face surface 1

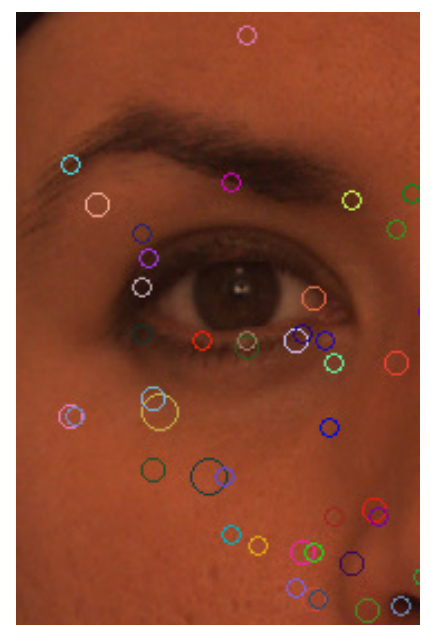

(b) Detailed view of face surface 2

Fig. 6: Detailed view of two faces with neutral expression. Corresponding scale space extrema are shown in the same color.

\section{A. Pose Normalisation}

Because the angle at which a person is scanned can not always be determined at scan time, also 3D face scans show variation because of the head pose. This is mostly the first correction that has to be made in $3 \mathrm{D}$ face preprocessing. Because of the 3D nature of the face scans, face pose normalisation comes down to the determination of a rigid transformation matrix. In this experiment, we do this by matching meshSIFT features between the faces that need to be face pose normalised. To eliminate the contribution of possible false matches, the determination of the rigid transformation matrix is performed using the RANdom SAmple Consensus (RANSAC) algorithm [21], a non-deterministic iterative algorithm to estimate parameters of a mathematical model based on data containing outliers.

For the validation of the pose normalisations, we made use of the landmarks that are available in the Bosphorus database (see figure 7). We consider the mean distance between corresponding landmarks after pose normalisation. Because these distances also incorporate deviations due to inter-person and expression variations, we consider a pose normalisation as correct if all Euclidean landmark distances after pose normalisation are smaller than $20 \mathrm{~mm}$. This distance was determined empirically. Table I summarizes the results of the pose normalisation experiment. Because of the fact that yaw rotations of $+90^{\circ}$ and $-90^{\circ}$ can lead to the physical impossibility of keypoint matches, we also show the results of the pose normalisation experiment without taking these scans into account. Because we noticed that the scans with yaw rotations of $+45^{\circ}$ and $-45^{\circ}$ are sometimes of bad quality, the results without taking these scans into account are displayed as well. To show the influence of the expressions, also results when only taking frontal neutral expression scans are shown.

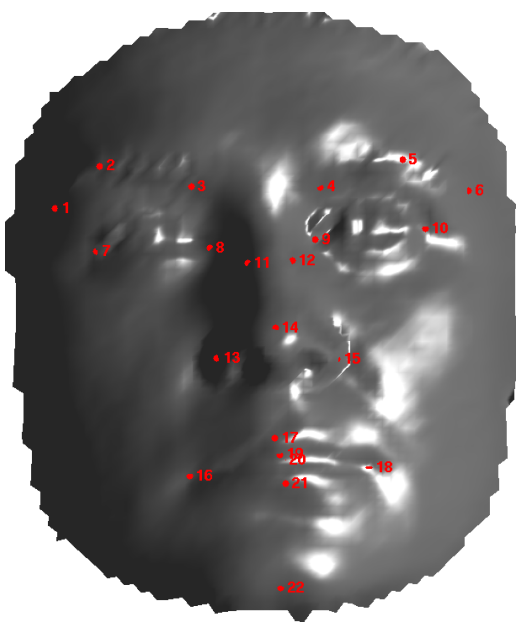

Fig. 7: 22 manually indicated landmarks, available with the Bosphorus database. Two additional landmarks are situated at the ear base and are thus not visible in this image.

TABLE I: Results of the pose normalisation experiment.

\begin{tabular}{|c||c|c|}
\hline & mean dist. (mm) & $\%$ correct \\
\hline All meshes & 6.28 & 83.73 \\
\hline All meshes except $\pm 90^{\circ}$ & 6.22 & 87.65 \\
\hline All meshes except $\pm 90^{\circ}$ and $\pm 45^{\circ}$ & 5.86 & 90.25 \\
\hline Only neutral face expressions & 3.95 & 98.32 \\
\hline
\end{tabular}

\section{B. Face Recognition}

To perform a face recognition experiment on the Bosphorus database using meshSIFT, we implement a Bag Of Features approach: the similarity measure between two 3D face scans is defined as the number of matching features. We performed an identification experiment where the probe scans are compared to a gallery consisting of one neutral face scan of each of the different persons in the database. This experiment stands for the situation in which we have to identify an unknown individual. The identification only succeeds if, when given a face scan, the algorithm selects the gallery face scan belonging to the same person as the given face scan. The Rank 1 Recognition Rate (R1RR) and Rank 5 Recognition Rate (R5RR) are tabulated in table II. Because of the same reasons as with the pose normalisation experiment, we also tabulated results when omitting the $+90^{\circ}$ and $-90^{\circ}$, and both $+90^{\circ}$ and $-90^{\circ}$ and $+45^{\circ}$ and $-45^{\circ}$ yaw rotations, respectively. Also results when omitting also cross rotations (approximately $20^{\circ}$ pitch and $45^{\circ}$ yaw rotation, and approximately $-20^{\circ}$ pitch and $45^{\circ}$ yaw rotation, respectively, are included in the Bosphorus database) are tabulated.

We also performed a verification experiment, where the faces in the database are pairwise compared. This experiment stands for the situation in which we have to to verify that the individual is who he claims to be. Based on one threshold, the algorithm has to decide whether two face scans are from the same person. Based on this experiment, the Equal Error Rate (EER) can be computed. If the threshold 
TABLE II: Results for the face identification experiment

\begin{tabular}{|c||c|c|}
\hline & R1RR (\%) & R5RR (\%) \\
\hline All meshes & 93.66 & 96.62 \\
\hline All meshes except $\pm 90^{\circ}$ & 97.01 & 99.31 \\
\hline All meshes except $\pm 90^{\circ}$ and $\pm 45^{\circ}$ & 97.59 & 99.44 \\
\hline $\begin{array}{c}\text { All meshes except } \pm 90^{\circ}, \pm 45^{\circ}, \\
\pm 30^{\circ} \text { and cross rotations }\end{array}$ & 97.73 & 99.42 \\
\hline
\end{tabular}

value is set so that the false acceptance rate and its false rejection rate are equal, the common value is referred to as the EER. The lower the EER, the higher the accuracy of the algorithm. The results are shown in III, both for the all to all (ata) and the all to neutral (atn) scenario. The latter has the advantage that comparisons of, for instance, scans with $+90^{\circ}$ and $-90^{\circ}$ yaw rotations are avoided.

TABLE III: Results for the face authentication experiment

\begin{tabular}{|c||c|c|}
\hline & EER $\operatorname{ata}(\%)$ & EER $\operatorname{atn}(\%)$ \\
\hline All meshes & 19.52 & 10.75 \\
\hline All meshes except $90^{\circ}$ & 14.96 & 7.92 \\
\hline All meshes except 90 and $45^{\circ}$ & 11.95 & 6.41 \\
\hline $\begin{array}{c}\text { All meshes except } 90,45, \\
30^{\circ} \text { and cross rotations }\end{array}$ & 9.45 & 5.08 \\
\hline
\end{tabular}

The reason that the EER is relatively poor in comparison to the excellent R1RR, is the nature of the similarity measure. The similarity we use is the number of corresponding features between two 3D face scans. We do not normalize for overlap area, noise ration ... For a better EER, we will have to design a new similarity measure that is invariant to these effects.

To give an indication of the number of scale-space extrema, and the number of correspondences retrieved, we tabulated the mean number of scale-space extrema, matches at rank 1 and matches at rank 5 for all face scans in the Bosphorus databse in table IV.

\section{DISCUSSION}

Important advantages of using meshSIFT for pose normalisation are the robustness against missing data, large rotations and translations, as occuring in the Bosphours database. This is of great importance for robust automatic initialization before fine registration.

The use of meshSIFT for 3D face recognition is a natural way to compare faces based on characteristic features in the human face [20]. It also allows face comparisons even when parts of the face are occluded.

The main shortcoming of meshSIFT for face recognition is that the subject's global shape characteristics are not taken into account during comparison. Another disadvantage is the rather high computation time, mainly for constructing the local shape descriptor. Large rotations and expression variations decrease the number of matches between feature vectors and thus the recognition performance. A more advanced similarity measure based on the angles between the feature vectors or other score-based measures could solve this issue.

It is interesting to compare the obtained recognition results with other algorithms that have been published and validated on the same database. The results of this comparison are shown in Table V. Remark that only the method presented in this paper was validated on the whole database. The methods described in [22] and [23] both perform face recognition on regions that are more robust to expression variations (mainly the nose region). In [24], two benchmark algorithms are implemented and validated, namely the Iterative Closest Point algorithm and a method based on Principal Component Analyis of depth images.

TABLE V: Comparison with other methods mentioned in literature, validated on a subset of the Bosphorus database.

\begin{tabular}{|l||c|c|c|}
\hline Method & R1RR & \# probes & gallery \\
\hline $\begin{array}{l}\text { ours } \\
\text { frontal } \\
\text { all }\end{array}$ & $97.7 \%$ & 3186 & 105 \\
\hline $\begin{array}{l}\text { Alyüz et al. [22] } \\
\text { all }\end{array}$ & $93.7 \%$ & 4561 & 105 \\
\hline $\begin{array}{l}\text { Dibeklioğlu et al. [23] } \\
\text { frontal }\end{array}$ & $95.3 \%$ & 1508 & 47 \\
$\quad$ rotations & $89.2 \%$ & 1527 & 47 \\
\hline $\begin{array}{c}\text { Alyüz et al. [24] } \\
\text { all (ICP) }\end{array}$ & $62.6 \%$ & 423 & 47 \\
all (PCA) & $72.4 \%$ & 1508 & 47 \\
& $70.6 \%$ & 1508 & 47 \\
\hline
\end{tabular}

Numerical comparison has to be done with sufficient caution since the subsets differ for the different methods. However, we still observe an excellent performance of our method described here, compared to the methods in the literature.

\section{CONCLUSION AND FUTURE WORK}

In this paper, we present an adapted version of the SIFTalgorithm [1] for meshes (called meshSIFT). This algorithm allows reliable detection of scale space extrema and a scale and orientation invariant description in a feature vector. The matches, found with this feature vector, open possibilities for pose normalisation and face recognition. It is possible to normalize the pose of $90 \%$ of the face surfaces in the Bosphorus database $\left(90^{\circ}\right.$ and $45^{\circ}$ rotations excluded). The described algorithm correctly identifies $97.0 \%$ of the face surfaces in the Bosphorus database ( $90^{\circ}$ rotations excluded). In the verification scenario, an equal error rate of $15.0 \%$ up to $5.1 \%$ is possible (depending on the face surfaces considered).

As future work, we propose to examine other criteria to define matches between feature vectors. Moreover, a more advanced similarity measure (instead of the number of matches) could also improve 3D face recognition performance.

\section{ACKNOWLEDGMENTS}

This work is supported by the Flemish Institute for the Promotion of Innovation by Science and Technology in 
TABLE IV: Number of scale space extrema and matches

\begin{tabular}{|c||c|c|c|}
\hline & \# scale-space extrema & \# matches @ rank 1 & \# matches @ rank 5 \\
\hline mean & 559.6 & 97.3 & 69.2 \\
\hline standard deviation & 86.6 & 43.7 & 33.3 \\
\hline
\end{tabular}

Flanders (IWT Vlaanderen), the Research Programme of the Fund for Scientific Research - Flanders (Belgium) (FWO) and the Research Fund K.U.Leuven.

\section{REFERENCES}

[1] D. G. Lowe, "Distinctive image features from scale-invariant keypoints," Int. J. Comput. Vision, vol. 60, no. 2, pp. 91-110, 2004.

[2] A. Savran, N. Alyuz, H. Dibeklioglu, O. Celiktutan, B. Gokberk, B. Sankur, and L. Akarun, "Bosphorus database for 3D face analysis," in The First COST 2101 Workshop on Biometrics and Identity Management (BIOD 2008), (Denmark), May 2008. Roskilde University.

[3] M. Brown and D. Lowe, "Invariant Features from Interest Point Groups," in Proceedings of the 13th British Machine Vision Conference, (Cardiff), pp. 253-262, 2002.

[4] M. Bicego, A. Lagorio, E. Grosso, and M. Tistarelli, "On the use of SIFT features for face authentication," in Computer Vision and Pattern Recognition Workshop, 2006. CVPRW '06. Conference on, pp. 35 35, 17-22 2006.

[5] D. Kisku, A. Rattani, E. Grosso, and M. Tistarelli, "Face identification by SIFT-based complete graph topology," in Automatic Identification Advanced Technologies, 2007 IEEE Workshop on, pp. 63 -68, 7-8 2007.

[6] W. Cheung and G. Hamarneh, "N-SIFT: N-dimensional scale invariant feature transform for matching medical images," in Biomedical Imaging: From Nano to Macro, 2007. ISBI 2007. 4th IEEE International Symposium on, pp. $720-723,12-152007$.

[7] K. Osada, T. Furuya, and R. Ohbuchi, "SHREC'08 entry: Local volumetric features for 3D model retrieval," in Shape Modeling and Applications, 2008. SMI 2008. IEEE International Conference on, pp. $245-246,4-62008$

[8] T.-W. R. Lo and J. P. Siebert, "Local feature extraction and matching on range images: 2.5D SIFT," Computer Vision and Image Understanding, vol. 113 , no. 12 , pp. $1235-1250,2009$. Special issue on 3D Representation for Object and Scene Recognition.

[9] A. Zaharescu, E. Boyer, K. Varanasi, and R. P. Horaud, "Surface feature detection and description with applications to mesh matching," in Proceedings of the IEEE Conference on Computer Vision and Pattern Recognition, (Miami Beach, Florida), June 2009.

[10] G. Zou, J. Hua, M. Dong, and H. Qin, "Surface matching with salient keypoints in geodesic scale space," Comput. Animat. Virtual Worlds, vol. 19, no. 3-4, pp. 399-410, 2008.

[11] A. Johnson, Spin-Images: A Representation for 3-D Surface Matching. $\mathrm{PhD}$ thesis, Robotics Institute, Carnegie Mellon University, Pittsburgh, PA, August 1997.

[12] U. Castellani, M. Cristani, S. Fantoni, and V. Murino, "Sparse points matching by combining 3D mesh saliency with statistical descriptors," Computer Graphics Forum, vol. 27, pp. 643-652, April 2008.

[13] W. Zhao, R. Chellappa, P. J. Phillips, and A. Rosenfeld, "Face recognition: A literature survey," ACM Comput. Surv., vol. 35, no. 4, pp. 399-458, 2003.

[14] K. W. Bowyer, K. Chang, and P. Flynn, "A survey of approaches and challenges in 3D and multi-modal 3D $+2 \mathrm{D}$ face recognition," Comput. Vis. Image Underst., vol. 101, no. 1, pp. 1-15, 2006.

[15] A. Mian, M. Bennamoun, and R. Owens, "Face recognition using 2D and 3D multimodal local features," pp. I: 860-870, 2006.

[16] A. Mian, M. Bennamoun, and R. Owens, "An efficient multimodal 2D3D hybrid approach to automatic face recognition," Pattern Analysis and Machine Intelligence, IEEE Transactions on, vol. 29, pp. 1927 -1943 , nov. 2007.

[17] K. G. Derpanis, "Overview of binomial filters." http: //www.cse.yorku.ca/ kosta/CompVis_Notes/ binomial_filters.pdf, March 2005.

[18] G. Peyre, "Toolbox graph.” MATLAB Central File Exchange Select, 2009.

[19] G. Peyre, "Toolbox mast marching." MATLAB Central File Exchange Select, 2009.
[20] D. Lowe, "Object recognition from local scale-invariant features," in Computer Vision, 1999. The Proceedings of the Seventh IEEE International Conference on, vol. 2, pp. 1150 -1157 vol.2, 1999.

[21] M. A. Fischler and R. C. Bolles, "Random sample consensus: a paradigm for model fitting with applications to image analysis and automated cartography," Commun. ACM, vol. 24, no. 6, pp. 381-395, 1981.

[22] N. Alyüz, B. Gökberk, and L. Akarun, "A 3D face recognition system for expression and occlusion invariance," in BTAS '08: Proceedings of the IEEE Second International Conference on Biometrics Theory, Applications and Systems, (Arlington, Virginia, USA), September 2008.

[23] H. Dibeklioğlu, B. Gökberk, and L. Akarun, "Nasal region-based 3D face recognition under pose and expression variations," in ICB '09: Proceedings of the Third International Conference on Advances in Biometrics, (Berlin, Heidelberg), pp. 309-318, Springer-Verlag, 2009.

[24] N. Alyüz, B. Gökberk, H. Dibeklioğlu, A. Savran, A. A. Salah, L. Akarun, and B. Sankur, "3D face recognition benchmarks on the bosphorus database with focus on facial expressions," pp. 57-66, 2008. 